\title{
A Resistance-Integral Natural-Coordinate Method for Diffusive Transport
}

\author{
T. N. Narasimhan
}

Received: 16 March 2009 / Accepted: 21 February 2010 / Published online: 10 March 2010

(C) The Author(s) 2010. This article is published with open access at Springerlink.com

\begin{abstract}
Numerical models of diffusive transport, commonly considered as approximate solvers of the partial differential equation, focus on evaluation of gradients at a point. Gradient-approximation inaccuracies manifest themselves as errors in conductances and capacitances that enter into matrices that are finally solved. In turn, these errors arise from a lack of consideration of flow geometry in the point's vicinity. In order to improve accuracy, flow geometry may be incorporated into evaluation of conductances and capacitances by choosing segments of flow tubes as volume elements. Flow tubes, inherent in the initial conditions, can be generated using appropriate interpolation schemes that are built into contouring algorithms. The principal task is to structure contouring tools in such a way as to generate information to facilitate accurate evaluation of conductances and capacitances. The logical framework of this novel approach is founded on the contributions of Maxwell, who visualized a flow domain as a collection of flow tubes, Ohm, who introduced the notion of resistance of a finite body, and Fick, who introduced a differential equation for diffusive flow through a tube of non-uniform cross section. Computer-graphics capability to implement this approach has become available only over the past two decades. Casting aside the notion of a point, the new paradigm is to evaluate flow-resistance over finite distances. The method suggested is referred to as a Resistance-Integral Natural-Coordinate Method (RINC). Formulated from first principles, this method dispenses with the differential equation as an intermediary to formulate the numerical equations. It proceeds directly from the physical problem to its numerical representation.
\end{abstract}

Keywords Numerical method - Natural-coordinates · Grid generator · Mesh generator · Integral methods

T. N. Narasimhan $(\bowtie)$

Department of Materials Science and Engineering, University of California, 210 Hearst Memorial Mining Building, Berkeley, CA 94720-1760, USA

e-mail: tnnarasimhan@lbl.gov

T. N. Narasimhan

Department of Environmental Science, Policy and Management, University of California,

210 Hearst Memorial Mining Building, Berkeley, CA 94720-1760, USA 


\section{Introduction}

Numerical models based on finite difference, finite element, integral finite difference, and finite volume methods are widely used to solve diffusion-type problems in porous media. These methods share a common philosophy of evaluating spatial gradient of a scalar potential (temperature, fluid pressure, hydraulic head, and chemical concentration) at a point using an approximating function, most commonly a linear function. These methods are precise when local flow geometry in the vicinity of a point of interest is linear, characterized by parallel flow lines. However, when local flow pattern deviates from linearity, or when the governing differential equation is nonlinear, approximation errors are introduced into the solution. Such errors arise from inaccuracies in the estimation of conductances between interconnected nodes or volume elements, and capacitances of individual volume elements. Commonly, these are referred to as truncation errors.

The fact that these errors arise when local flow patterns deviate from linearity suggests that consideration of local flow geometry in flux estimation can help reduce these errors. Accordingly, the premise of this study is that knowledge of local flow geometry inherent in the initial conditions of an initial-boundary-value problem can significantly help in improving accuracy and efficiency of numerical solutions.

The concepts central to this study are latent in the nineteenth century contributions of James Clerk Maxwell, Georg Simon Ohm, and Adolf Fick. However, what makes the new approach meaningful is that the necessary computational and graphics capabilities have become available only over the past 20 years. This article starts with a brief statement of the essential features of conventional numerical models for diffusive transport, followed by a description of the role of flow geometry in governing the magnitude of fluxes and magnitude of capacitance under transient conditions. Next, a new approach is presented in which focus is shifted away from points to volume elements that are defined to be segments of flow tubes. It is then shown how mass or energy conservation over such elements can lead to solutions of improved accuracy. This article concludes with a discussion of the advantages of the new method and additional work that needs to be done to make the method workable.

Collectively, integral finite difference method, finite volume method, and the finite element method can be considered as solvers of an integro-differential equation by disctretized methods. In comparison, the resistance-integral natural-coordinate method directly solves a self-consistent difference equation described in natural coordinates.

Diffusive transport occurs in general three dimensions. A small class of two-dimensional diffusion problems are amenable to treatment in terms of potential functions and stream functions based on Cauchy-Riemann conditions stemming from the theory of complex variables. However, the method presented in this study is developed independent of the differential equation. Therefore, potential functions and stream functions are not of direct relevance to this study.

For solving advective-diffusion transport problems, stream-line simulation methods are widely used in the literature. In these methods, contaminant praticles are transported along stream lines obtained by first solving the groundwater flow equation using conventional finite element or finite difference methods, and then contouring the resulting field of hydraulic head. The resistance-integral method presented in this study is for diffusive transport, and hence is not to be confused with these stream-line simulation methods intended for advective transport. 


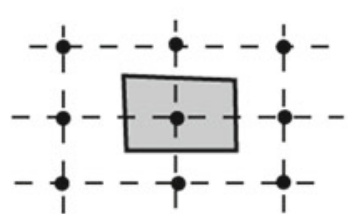

Finite Difference

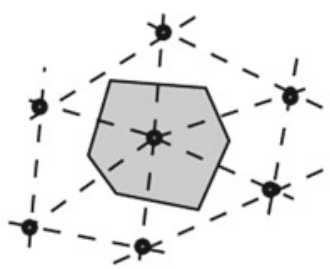

Integral Finite Difference Finite Volume

(Defined by perp. bisectors)

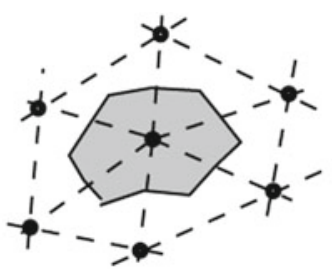

Finite Element (Defined by medians)

Fig. 1 Nodal points and associated volume elements in different numerical methods

\section{Conventional Numerical Methods}

Conventional numerical methods include finite difference, finite element, integral finite difference, and control volume methods. For all these methods, the starting point is the partial differential equation,

$$
\nabla K \cdot \nabla \varphi=c \frac{\partial \varphi}{\partial t},
$$

where, $K$ is conductivity (e.g., thermal, hydraulic), $c$ is volumetric specific capacitance (e.g., volumetric specific heat, specific storage of groundwater systems), $\varphi$ is a scalar potential (e.g., temperature, pressure, and hydraulic head), and $t$ is time. In numerical models, (1) is generally treated as being nonlinear in that $K$ may be a function of $\varphi$. In all these methods, a sufficiently large number of points (nodal points) are chosen to span the flow domain, and (1) is assumed to apply at each of these points in a discrete sense. Although a variety of mathematical techniques are used to derive the numerical equations, all these methods reduce, in the final analysis, to eliminating the second spatial derivative, and conserving mass or energy over appropriately defined volume elements around each nodal point. To this end, fluxes entering or leaving a volume element across its bounding surface segments are calculated by assuming that the spatial gradient at the surface is equal to linear slope of potential between the points on either side of the surface. The flux so calculated is accurate if flow lines in the vicinity of the surface are linear. Errors ensue if flow in the vicinity of the surface converges or diverges, characterized by non-parallel flow lines. The disposition of nodal points and associated volume elements is illustrated in Fig. 1.

The volumetric capacitance $C$ of an element is set equal to its volume times the specific capacity (e.g., volumetric specific heat, specific storage of groundwater systems). It is assumed that net accumulation over an element divided by $C$ gives the change in potential at the nodal point of the element. This assumption too is a source of error because the concept of specific heat, by definition, pertains to change in heat content from one thermostatic state to another thermostatic state. Under thermostatic conditions, temperature everywhere in an element is the same. Under transient conditions, temperature is spatially variable in the element. Therefore, to define a capacitance for a volume element that exists in a transient state, additional consideration is necessary.

The discretized conservation equation for an element $j$ takes the form,

$$
\Delta \varphi_{j}=\varphi_{j}\left(t^{0}+\Delta t\right)-\varphi_{j}\left(t^{0}\right)=\frac{\Delta t \sum_{m} U_{j, m}\left(\left\langle\varphi_{m}\right\rangle-\left\langle\varphi_{j}\right\rangle\right)}{C_{j}},
$$


where $\mathrm{U}_{j, m}$ is the conductance between nodes $j$ and $m, \mathrm{C}_{j}$ is the capacitance of node $j,\langle\varphi\rangle$ is a time- averaged value of $\varphi$ over the interval between $t^{0}$ and $t^{0}+\Delta t$. Commonly,

$$
\langle\varphi\rangle=\varphi\left(t^{0}+\lambda \Delta t\right),
$$

where $\mathrm{t}^{0}$ is time at the beginning of the time interval $\Delta t$, and $0<\lambda<1$ is a weighting factor. For stable solutions, $\lambda$ must be $>0.5$. When $\lambda=0$, (2) is an explicit relation, vulnerable to instability. For stable explicit calculation of potential for node $j$, the time step must satisfy the requirement (Narasimhan and Witherspoon 1976),

$$
(\Delta t)_{j} \leq \frac{C_{j}}{\sum_{m} U_{j, m}} .
$$

For $\lambda>0,(2)$ in conjunction with (3) leads to a set of linear equations that are solved using matrix solvers. Accuracy of solutions increases with finer grid discretization.

\section{Role of Geometry in Evaluating Flux}

Although linear flux laws are often expressed in terms of a gradient of potential at a point, the gradient-based approach is of limited value when dealing with observational data. Observational data invariably consist of measured values of potential at discrete locations. Given the values of potential at two points separated by a distance, it is not possible to know the gradient of potential in the space between the points, except in the special and trivial case of parallel flow lines, with $K$ independent of $\varphi$. In this specific case, gradient at any location between the points is exactly equal to the magnitude of the uniform slope of the potential between the points. Even in the case of parallel flow lines, if $K$ is dependent on $\varphi$, gradient can be determined exactly only when $K$ has an exponential dependence on $\varphi$. In general, therefore, when flow lines converge and diverge, and/or when $K$ depends on $\varphi$, gradient cannot be evaluated with precision without much additional information and effort. Accordingly, there is justification to abandon the traditional gradient-based approach in favor of a more effective alternative. Such an alternative is available in Ohm's concept of resistance. Although inspired by Fourier's differential equation, Ohm (1827) found it practical to express a flux law for electric current in terms of difference in potential and resistance, rather than using a gradient. Generically, Ohm's Law states that flux through a flow tube equals the difference in potential between inlet and outlet divided by the resistance offered to flow by the material body.

Now consider a flow tube of non-uniform cross section as shown in Fig. 2, bounded at inlet and outlet by isopotential surfaces. A flow line, whose length is equal to the arithmetic average of all flow lines is chosen as a curvilinear $X$-axis. Within this flow tube, $\mathrm{A}(x)$ denotes the area of the isopotential surface at $x$. Under these conditions, Ohm's law leads to,

$$
Q=\frac{\varphi_{\text {in }}-\varphi_{\text {out }}}{R_{\text {in }, \text { out }}}=\frac{\varphi_{\text {in }}-\varphi_{\text {out }}}{\int_{X_{\text {in }}}^{x_{\text {out }}} \frac{\mathrm{d} y}{K(y) A(y)}},
$$

where $Q$ is flux, and $R$ is resistance. The subscripts in and out denote inlet and outlet. This shows that resistance is a function of the material property (conductivity, $K$ ) and the shape of the flow tube (geometry of flow). The variation of A with position along the curvilinear $X$ axis quantifies the geometric information needed to evaluate the integral.

Evaluation of flux according to (5) becomes more involved when conductivity is a function of $\varphi$; i.e., when the governing differential equation is nonlinear. In this case, $K$ is only known as a function of $\varphi$, while the integral in (5) is defined in space. Therefore, it is necessary to 
Fig. 2 Flow tube with variable cross section along flow path

\section{A non-uniform flow tube}

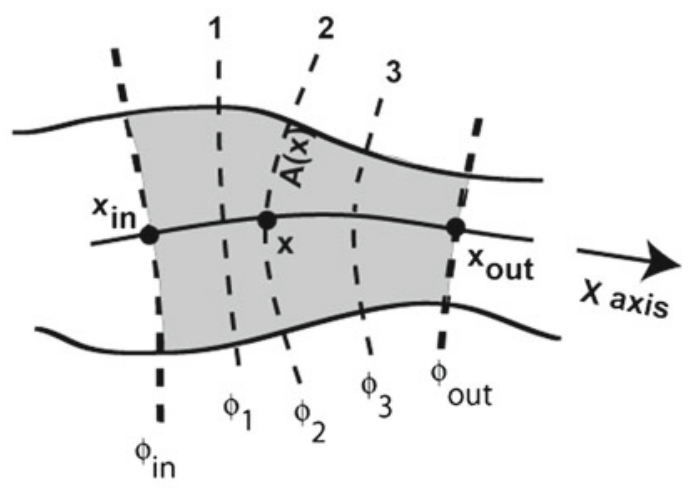

$A(x)$ is area of cross section of ispotential at $x$ 1,2 , and 3 denote three isopotentials

cast the integral in the $\varphi$-domain. Thus, multiplying and dividing the integrand by $\mathrm{d} \varphi$, we get,

$$
Q=\frac{\varphi_{\text {in }}-\varphi_{\text {out }}}{\int_{\varphi_{\text {in }}}^{\varphi_{\text {out }}} \frac{\mathrm{d} \varphi}{K(\varphi) \frac{\mathrm{d}_{\varphi}}{\mathrm{d}_{y}} A(\varphi)}},
$$

Since $\mathrm{d} \varphi / \mathrm{d} y$ is not known a priori in the integrand of (6), $Q$ and resistance are implicitly related to each other. They have to be evaluated together through an iterative process. Conventional assumption that flux can be explicitly calculated is not credible anymore.

In view of (5) and (6), steady-state flux through a flow tube of non-uniform cross section may be expressed as,

$$
Q=U\left(\varphi_{\text {in }}-\varphi_{\text {out }}\right)
$$

where $U=\left(1 / R_{\text {in,out }}\right)$ is the conductance of the tube. Given the definition of conductivity, (7) constitutes perhaps the most precise way of calculating flux.

\section{Role of Geometry in Calculating Capacitance}

The concept of specific heat was introduced by Lavoisier and Laplace (1783) when they invented the ice calorimeter and measured the capacity of materials to hold heat in comparison with that of water. By definition, specific heat is the quantity of heat required to raise the temperature of a unit mass of a material by one degree from one thermostatic state to another. When the mass of a body differs from unity, its capacity to absorb heat is referred to as its "heat capacity". In the context of the heat equation, one uses volumetric specific heat and volumetric heat capacity, which equals specific heat times mass density.

The specification of thermostatic state in the definition is important. In a well-stirred calorimeter, the temperature everywhere within the body is the same. Thus, the change in temperature is a unique number, and specific heat is an unambiguous quantity.

Now consider a body which is not under thermal equilibrium, and temperature is spatially variable within the body. In this case, when a certain quantity of heat is added to the body, 
Fig. 3 Capacitance of a transient volume element

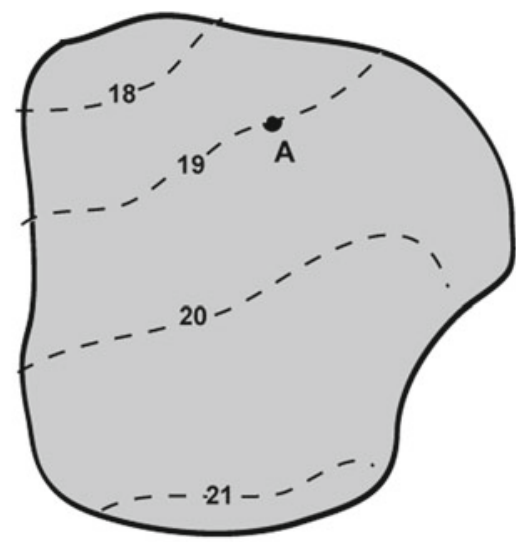

A transient volume element with contours of isotherms

the magnitude of temperature change will be spatially variable. Clearly, one cannot apply the concept of specific heat or heat capacity in this case. Thus, to extend the concept of heat capacity to a body under transient conditions, it is necessary to introduce further assumptions.

Accordingly, consider a body that is relatively large in size over which temperature is spatially variable (Fig. 3). Within this, consider a volume element that is large enough so that microscopic local fluctuations are smoothed out, and macroscopic observations with instruments are possible. Over this elemental volume $\mathrm{d} V$, we will assume local thermostatic conditions, so that the concept of specific heat is applicable. Under these assumptions, we may obtain the heat content of the body at an instant in time by integrating $(\rho c T \mathrm{~d} V)$ over the body. Now, since $T$ is spatially variable, heat capacity for this body is not well-defined. However, we may define an operational heat capacity as a function of position. Thus, if temperature at $\mathrm{A}$ is $T_{\mathrm{A}}$, the heat capacity associated with $\mathrm{A}$ is,

$$
C_{\mathrm{A}}=\int_{V} \frac{\rho c T \mathrm{~d} V}{T_{\mathrm{A}}},
$$

where $C_{\mathrm{A}}$ is the appropriate heat capacity. Clearly, $C_{\mathrm{A}}$ will be constant at all points on the isothermal surface on which A lies.

An alternate way is to assume that the capacitance of the element is associated with an average temperature $\langle T\rangle$ and is given by, $C=V \rho \mathrm{c}$. Then, the average temperature over the element is given by,

$$
\langle T\rangle=\frac{\int_{V} \rho c T \mathrm{~d} V}{C} .
$$

Given this, the desired point can lie anywhere on the isothermal contour with magnitude $\langle T\rangle$. $C$ is associated with this isothermal contour. This has been termed "location of average" by Narasimhan (1985).

\section{Resistance-Integral Method for Modeling Diffusive Transport}

In view of the concepts presented above, it is now possible to outline a novel modeling approach centered around evaluating the resistance integral along curvilinear flow paths. In 
A

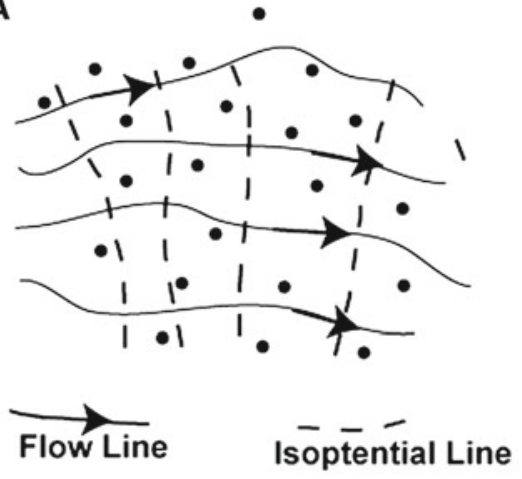

- Data Point

\section{Contours generated from a given set of initial conditions}

B
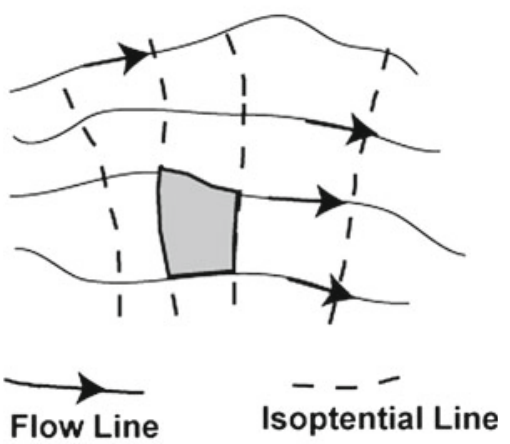

Fig. 4 Contour map based on initial conditions (a), and a segment of flow tube as a volume element (b)

this approach, one starts by choosing a volume element over which mass or energy can be conserved, and conveniently chooses a nodal point within the element by suitably defining a capacitance associated with that point. Whereas conventional numerical models start with a point and mathematically define a volume element around them (Fig. 1), the new approach reverses the process by starting with a volume element, and then associating a nodal point within it.

In 1855, Maxwell visualized the steady flow of an incompressible fluid in a resistive medium in terms of a collection of flow tubes with associated orthogonal isopotential surfaces (Maxwell 1864; Narasimhan 2003). Rather than starting with the differential equation, Maxwell took a dynamical approach, and founded his visualization on a series of postulates related to balancing of impelling and resistive forces. Contemporaneously, Fick (1855) proposed, for molecular diffusion, a differential equation accounting for variable cross section along flow path. Thus,

$$
K\left[\frac{\mathrm{d}^{2} c}{\mathrm{~d} x^{2}}+\frac{1}{A} \frac{\mathrm{d} A}{\mathrm{~d} x} \frac{\mathrm{d} c}{\mathrm{~d} x}\right]=0,
$$

where $c$ is concentration. It can be shown that when $K$ is independent of $c$, the exact solution to (10) is given by (5).

The contributions of Maxwell, Ohm, and Fick provide a framework for a novel way of modeling diffusive transport by discretizing the flow domain into volume elements that are segments of flow tubes. The premise is that given such an element, one can accurately evaluate conductances according to (5) or (6), and capacitances according to (8).

Let, as shown in Fig. 4a, data on potentials be provided at a finite number of points at time $t=0$. Since empirically established flux laws (Fourier, Ohm, Darcy, and Fick) are only valid for steady-state flow, and in order that these laws may be applied with credibility, assume that these laws are applicable locally over the flow domain, although the global system is transient. Using this assumption, the first step is to prepare, using an appropriate interpolation scheme, a convenient number of isopotential surfaces and associated flow lines. For this purpose, one may use interpolation schemes that are consistent with diffusive flux 


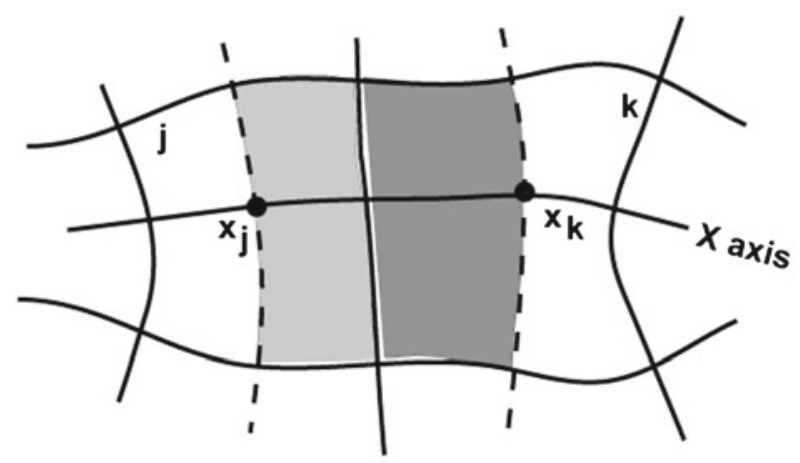

Conductance between elements $\mathrm{j}$ and $\mathrm{k}$

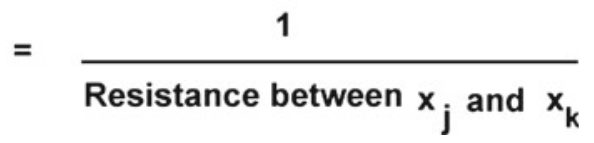

Fig. 5 Flow tube segment between isopotentials at $x_{j}$ and $x_{k}$

laws, or may use an energy minimization rationale (variational method). This results in the flow domain being entirely pervaded by flow tubes. Segments of these flow tubes now constitute the computational grid for numerical simulation. As shown in Fig. 4b, each flow-tube segment becomes a volume element over which mass or energy is to be conserved.

The next step is to choose a nodal point within each element. As has been established already, under transient conditions, the capacitance of a volume element is a function of position within the element. Consequently, any point within an element as shown in Fig. $4 \mathrm{~b}$ could be chosen as a nodal point, provided the appropriate capacitance is suitably calculated. The manner in which a nodal point may be chosen within a volume element is apparent in Fig. 2. The point of intersection of the $X$ axis with a conveniently chosen isopotential surface is the desired nodal point.

Since a volume element is a segment of a flow tube, it has an upstream surface of inflow and a down stream surface of outflow, with no flow across the other surfaces. The procedure for evaluating conductance between adjoining elements $j$ and $k$ is illustrated in Fig. 5. The region between the isotherms at $\mathrm{x}_{j}$ and $\mathrm{x}_{k}$ is a flow tube. Assuming that the variation of $\mathrm{A}$ as a function of $\mathrm{x}$ for this segment can be generated from the contouring algorithms, the resistance $\mathrm{R}_{j, k}$ may obtained by evaluating integral (5) when $K$ is independent of $\varphi$, or (6) when $K$ is dependent on $\varphi$. The required conductance is the reciprocal of $\mathrm{R}_{j, k}$.

In view of the foregoing, the conservation equation for an element $\mathrm{j}$ is,

$$
(\Delta \varphi)_{j}=\frac{\Delta t \sum_{m=j, k} U_{j, m}\left(\left\langle\varphi_{m}\right\rangle-\left\langle\varphi_{j}\right\rangle\right)}{C_{j}}
$$

where $\langle\varphi\rangle$ is as defined in (3). For stable solutions using large time steps, $0.5<\lambda<1$, and (11) leads to set of linear equations that need to be solved using matrix solvers.

During the 1960s, when numerical models came into prominence, the computers were slow. Consequently, explicit solutions using $\lambda=0$ were inefficient due to the very large number of time steps needed to advance in time. It was, therefore, very advantageous to use matrix solvers that enabled the use of large time steps. 


\section{An Illustration}

In an attempt to find ways of solving transient subsurface flow problems independently of the differential equation, Narasimhan (1985) introduced the notion of imbedding geometry in Darcy's Law, and the notion of associating the average value of potential over a volume element with a particular location within the element. He demonstrated how these two notions helped greatly in evaluating the conductance between adjoining volume elements, and the estimation average changes in potential over an element during an interval of time. For the case of conductivity independent of $\varphi$, he used (5) to calculate resistance between adjoining volume elements, and for location of average, he essentially used (8). In order to check the efficacy of these concepts, he considered the problem of transient radial flow of water to a fully penetrating well of finite radius, pumping at a steady volumetric rate $Q$ from an aquifer of constant thickness $H$.

In order to numerically simulate this system using the input format of an integral finite difference algorithm (Narasimhan and Witherspoon 1976), the flow domain extending from a radius of 0.1-625,000 m was discretized into 4 volume elements as shown in Fig. 6a. The nodal points for the four elements were chosen at distances of 3.04, 151.9, 7,593, and $379,625 \mathrm{~m}$ from the axis of the well. The numerical solution (solid dots) is shown compared with analytical solution (with well-bore storage effects) in Fig. 6b with dimensionless time on the abcissa, and dimensionless pressure drawdown on the ordinate. The open circles indicate solution obtained with a conventional integral finite difference scheme. The curve representing theis solution pertains to a line-source, or equivalently, a well of vanishingly small radius. As can be seen, the method accounting for flow geometry has yielded surprisingly accurate results despite the extremely coarse discretization.

It could be argued that a finite element algorithm in radial coordinates would yield results of accuracy similar to what is shown in Fig. 6. The argument is valid and reinforces the premise of this study that incorporating geometry into the equation of motion can dramatically improve accuracy. However, there is a limitation with the finite element approach. The matrix of finite element equations must be prepared for each symmetry of interest. Such matrices, for example, can be prepared for cylindrical or spherical symmetries. However, it is not possible to prepare such matrices for flow systems of arbitrary geometry, without any noticeable symmetry. It is in regard to such flow systems of arbitrary geometry that the approach described in this study based on evaluating resistance using natural coordinates is powerful.

\section{Advantages of the New Approach}

The new approach outlined in the foregoing offers some philosophical and practical advantages. Philosophically, it dispenses entirely with the partial differential equation as an intermediary to develop numerical equations. Instead, it translates information directly from empirical observations to the discrete numerical model. This is an outcome of the recognition that steady-state flux depends on the nature of the resistive material as well as the geometry of the flow tube within which flow occurs. If due consideration can be given to flow tube geometry, then, accuracy is no more contingent on approximation errors associated with evaluating gradients. Second, very little consideration is normally bestowed on the role of capacitance in the diffusion process. Since the definition of specific heat is restricted to change in temperature from one thermostatic state to another, errors are inevitable if the concept is applied to volume elements in thermal disequilibrium. Such errors are avoided 
A
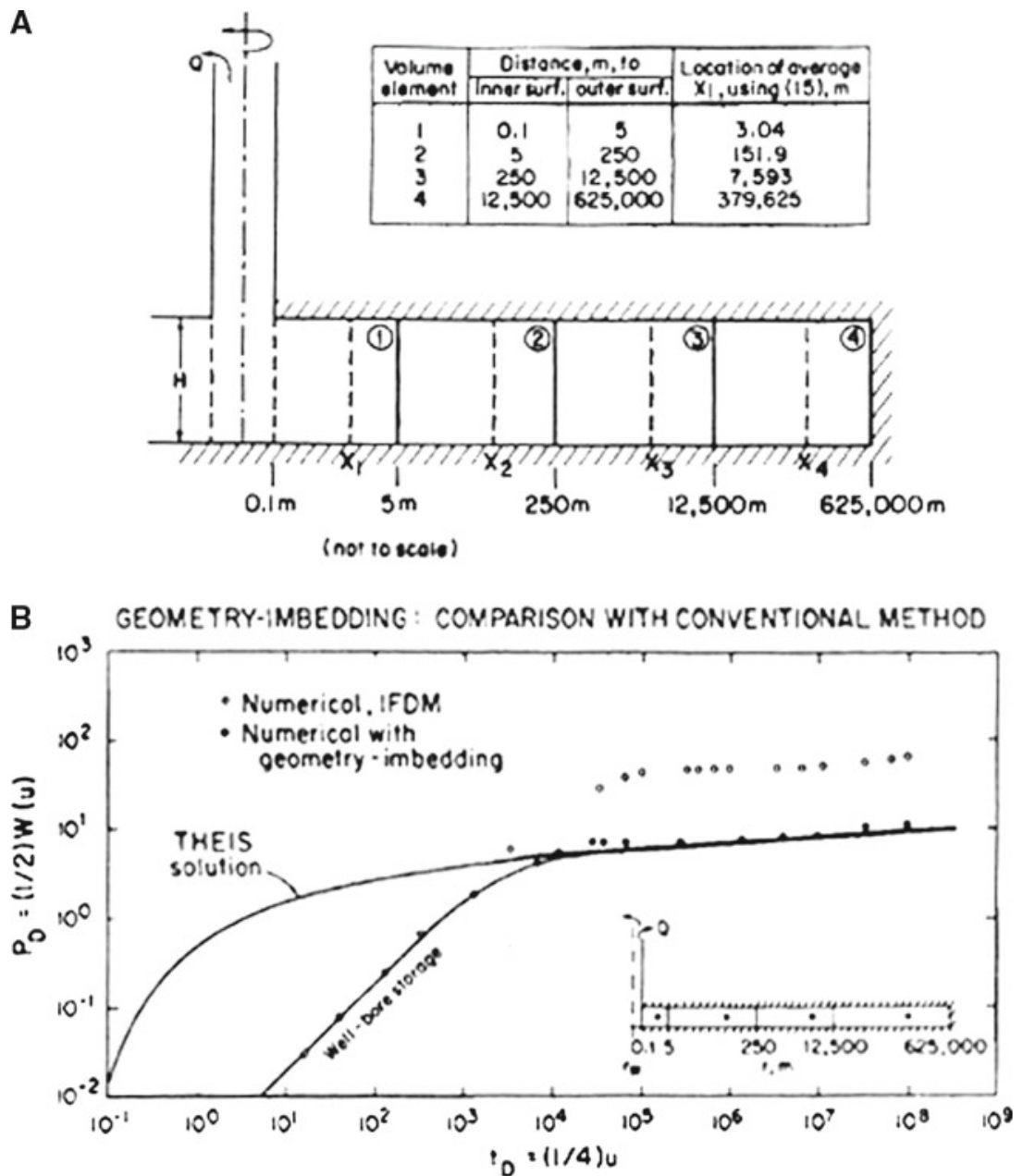

Fig. 6 Transient flow to a finite-radius well I an infinite aquifer. Comparison of RINC and conventional IFDM results

in the present study by making capacitance a function of position in an elemental volume. Finally, conventional perception that accuracy of the numerical solution increases with mesh refinement is no longer valid. It is possible to generate accurate solutions with coarse meshes as long as flow geometry is adequately accounted for.

On the practical side, consider first the stability criterion (4) for explicit calculation. Here, the magnitude of the numerator (capacitance) increases as volume increases, and the denominator (conductance) decreases with increasing separation between nodal points. In general, the magnitude of the stable time step increases as the square of the distance separating the nodal points. Thus, the coarser the grid, the large the stable time step. Second, explicit calculations for many elements can be carried out simultaneously, in parallel. Considering the ability of modern computers to carry out parallel processing, and the processing speed of modern computers, the explicit method of calculation becomes competitive with matrix methods. 
Apart from this computational advantage, there is an accuracy aspect that makes the explicit scheme very desirable. This relates to evaluating the resistance integral (6) in problems, such as unsaturated flow or multi-phase flow, in which conductivity is a function of potential. This integral has to be iteratively evaluated using the known values of $\varphi_{\text {in }}$ and $\varphi_{\text {out }}$ prevalent at beginning of the time step, $\mathrm{t}^{0}$. If one were to use time-averaging (i.e., use $0.5<\lambda \leq 1)$, then the use of the estimated time-averaged values of $\varphi_{\text {in }}$ and $\varphi_{\text {out }}$ will introduce additional errors. Consequently, the explicit scheme provides an added advantage of reducing errors in conductance and capacitance estimation in problems with $\varphi$-dependant material properties.

\section{What Needs to be Done}

The logical basis for the approach outlined above is simple. It has been known for over a century and half. However, its practical implementation has become a possibility only within the past two decades, with increasing power of digital computers, and availability of efficient graphics algorithms that produce contours of isopotentials and orthogonal flow lines. Three tasks lie ahead to make this approach a reality.

\subsection{Contouring Algorithms}

Presently, contouring algorithms are solely used for visualization purposes. A wealth of geometric information generated in the process of contouring calculations simply go unutilized. Lengths of curvilinear axes, areas of isopotential surfaces, and volumes of flow-tube segments are latent in such geometric information. In order to make full use of the information, contouring algorithms need to be made integral part of numerical models for diffusive transport. They need to be treated as grid generators in natural coordinates. It stands to reason that the computational grid, instead of being fixed, evolves in time as the flow pattern develops. How frequently the grid gets updated will be problem dependent.

That contouring algorithms can help significantly in improving the accuracy of numerical solutions provides an incentive to take a fresh look at the motivation underlying the development of contouring algorithms. Their value exceeds mere visualization. It is essential that the interpolation schemes of such algorithms are consistent with the underlying flux laws.

\subsection{Integration Modules}

Assuming that contouring-based grid generators provide the geometric details of flow tubes, the key computational task is to develop compact integration modules to iteratively evaluate the resistance integral (5) for $K$ independent of $\varphi$, and (6) for $K$ dependent on $\varphi$. These integrals are central to calculating conductances between volume elements, and for calculating capacitances of individual elements.

\subsection{Parallel Processing}

Finally, parallel processing powers of modern computers can be mobilized to handle time steps in novel ways. Currently, standard practice is to choose one time step for all volume elements in the system, and use matrix solvers to solve a set of linear equations. This need 
not be so if an explicit scheme is followed. In general, stable time steps for individual volume elements may vary widely over the flow domain. In such cases, parallel processing may help choose different time steps for different volume elements to efficiently progress in time.

\section{Concluding Remarks}

Newton's invention of the infinitesimal calculus profoundly influenced scientific thinking in terms of what happens at a point. In the case of diffusive transport, thinking inspired of fluxes as functions of gradient at points is a consequence of adherence to the differential equation. Unfortunately, gradient of potential at a point cannot be measured or observed. Gradient is an abstract concept. It has to be inferred from values of potential measured at discrete locations, separated by finite distances. Such inference is accurately possible only when the pattern of flow in the vicinity of the points is prescribed a priori.

Points and gradients are of enormous value in providing insights about classes of problems when they can be described algebraically, and when they have some inherent symmetry to facilitate analytic solutions (Narasimhan 2008). However, when a problem involves complex flow geometries and lack of noticeable symmetry, it needs to be described numerically and solved individually. Obtaining a solution for such a problem requires simultaneous, coupled consideration of flow geometry and associated spatial distribution of potentials. For such simultaneous consideration of geometry and potentials, the notions of flow tubes and resistance are more advantageous than those of points and gradients.

This study does not question the power of the partial differential equation to provide enormous insights into physical problems whenever they are amenable to obtaining analytical solutions. However, diffusive processes can also be described and solved using a global approach that is self-consistent and independent of the differential equation. Rather than points and gradients, the global approach depends on flow tubes and resistance. The global approach provides a natural connection between the observational world of physical data, and the discrete computational model, without requiring the partial differential equation as an intermediary. The resistance-integral natural-coordinate method takes advantage of this natural connection.

It is hoped that a researcher, or even a commercial enterprise with interest in numerical models, contouring, and computer graphics will take a serious interest in the novel approach suggested above. Potential rewards include a philosophical paradigm shift away from the differential equation to a physically meaningful approach to the numerical model, and a practical advantage of taking numerical model to the next level of computational sophistication.

Acknowledgments This study was supported partly by the Director, Office of Energy Research, Office of Basic Energy Sciences of the U.S. Department of Energy under Contract No. DE-AC03-76SF00098 through the Earth Sciences Division of Ernest Orlando Lawrence Berkeley National Laboratory, and partly by the Agricultural Extension Service, through the Division of Natural Resources, University of California.

Open Access This article is distributed under the terms of the Creative Commons Attribution Noncommercial License which permits any noncommercial use, distribution, and reproduction in any medium, provided the original author(s) and source are credited.

\section{References}

Fick, A.: On liquid diffusion. Phil. Mag. J. Sci. 10, 31-39 (1855) 
Lavoisier, M., Laplace, P.S.: Mémoire sur la Chaleur, de l'Académie Royale des Sciences, Paris, Paper read June 28 (1783). (English translation by H. Guerlac, Neale Watson Academic Publications Inc., New York (1982))

Maxwell, J.C.: On Faraday's lines of force, Read December 10, 1855 and February 11, 1856. Trans. Camb. Phil. Soc., 10, 27-83 (1864)

Narasimhan, T.N.: Geometry-imbedded Darcy's Law and transient subsurface flow. Water Resour. Res. 21(8), 1285-1292 (1985)

Narasimhan, T.N.: Maxwell, electromagnetism, and fluid flow in resistive media. EOS. Trans. Am. Geophys. Union 84(44), 411 (2003)

Narasimhan, T.N.: Laplace equation and Faraday's lines of force. Water Resour. Res. 44, W09412 (2008). doi:10.1029/2007/WR006221

Narasimhan, T.N., Witherspoon, P.A.: An integrated finite difference method for analyzing fluid flow in porous media. Water Resour. Res. 12(1), 57-64 (1976)

Ohm, G. S.: Die Galvanische Kette, Mathematisch Bearbeitet, Bei T.H. Riemann, Berlin (1827). (English translation by W. Francis, The Galvanic Circuit Investigated Mathematically, 269 p. Van Nostrand Co., New York (1891)) 